\title{
FISH physical mapping of 5S, 45S and Arabidopsis-type telomere sequence repeats in Chrysanthemum zawadskii showing intra-chromosomal variation and complexity in nature
}

\author{
Magdy Hussein Abd El-Twab ${ }^{1,2}$ and Katsuhiko Kondo ${ }^{1,3}$ \\ ${ }^{1}$ Laboratory of Plant Chromosome and Gene Stock, Graduate School of Science, Hiroshima \\ University, 1-4-3 Kagamiyama, Higashi-Hiroshima City 739-8524, Japan. \\ ${ }^{2}$ Botany Department, Faculty of Science, El-Minia University, El-Minia City, Egypt \\ ${ }^{3}$ Author for correspondence (kkondo@hiroshima-u.ac.jp) \\ Received April 7, 2005; accepted September 11, 2006
}

\begin{abstract}
Chrysanthemum zawadskii showed 2n=54 (hexaploid chromosme number) and extensive variation in the color of the ligules (from white to pinkish). FISH yellow colored signals of the 5S, 45S rDNA and telomeres were obtained after hybridized of the biotin labeled of the $5 \mathrm{~S}$ rDNA, pTa71 and telomeric sequence repeats probes to somatic chromosomes. They showed 5-6 interstitial and two terminal signals at the 5S rDNA region, 10-14 terminal and 4-5 interstitial signals at the 45S rDNA region and the telomeric signals at the region of telomeric sequence repeats on both chromosome arms in the chromosome complement. Bicolor FISH showed that co-localization of the $5 \mathrm{~S}$ and $45 \mathrm{~S}$ rDNA on two terminal and five interstitial loci. This phenomenon might indicate a natural hybridization with closely related genera and/or genome rearrangement.
\end{abstract}

KEYWORDS: Chrysanthemum zawadskii, FISH, 5S, 45S rDNA, telomere

Polyploidy has played an important role in the evolution of higher plants. Since $70 \%$ of all angiosperm species are of polyploid origin (Leich and Bennett 1997; Soltis and Soltis 2000). The widespread occurrence of polyploidy has been attributed to the potential of polyploid species to adapt to a wider range of habitats and survive better in unstable climates than their diploid progenitors; therefore, polyploid evolution has been a subject of intensive study for more than half a century. An appreciation of the types of changes in chromosomes/genomes that have occurred during species evolution, has a direct impact on the basic and applied botany and to the aims of plant researchers and breeders in understanding genome evolution and genetics makes this area worthy of further investigation (Heslop-Harrison 2000).

In nature, closely related taxa might make crosshybrids and perform a polyploid series, which plays an important role in the chromosome evolution of the species (Kondo et al. 1994). Several chromosome studies of Chinese and Japanese genera in narrow sense characterized by polyploid series, which were important to justify and clarify the cytogeographical disjunction and evolutionary process (Kondo et al. 1994, Abd El-Twab and Kondo 2003). Therefore, several chromosome analyses of Chrysanthemum and its allied genera have been carried out extensively in the Japanese and Chinese species since 1987 (e.g., Tanaka et al. 1989; Kondo et al. 1994, Abd El-Twab and Kondo 2003).

In higher eukaryotes, rDNA's are organized into two distinct gene classes of the major rDNA cluster encoding $45 \mathrm{~S}$ rDNAs; and the minor rDNA cluster encoding $5 \mathrm{~S}$ rDNA. The minor rDNAs are usually found in loci that are separate from those of the major rDNAs and, unlike major rDNA, the minor rDNA is not involved in the nucleolus formation (Inafuku 2000). Each of $10^{2}$ to $10^{4}$ 5S rDNA copies repeated in tandem comprises a highly conserved 120-nucleotid coding sequence and nontranscribed spacer (Inafuku 2000). The large ribosomal sub-unit contains a molecule of a 120 base 5S DNA (in all ribosomes except those of mitochondria) (Lewin 1997). The physical mapping of 5S rDNA was reported for the first time in Chrysanthemum species by Abd ElTwab and Kondo (2002).

In situ hybridization is a valuable method for studying the chromosomal distribution of DNA sequences and copy numbers at different sites, and to follow evolutionary changes in their physical organization in the genome (Harrison and Heslop-Harrison 1995). Fluorescence in situ hybridization (FISH) makes chromosomes of plant species providing the information of molecular characters of nucleolar organizing region (NORs) (Heslop-Harrison et al. 1991). NOR loci are chromosome regions most intensively studied, in terms of both their structure and function. Some molecular evidences indicated that $45 \mathrm{~S}$ rDNA is located on NOR-bearing chromosomes (Kondo et al. 1996; Ørgaard and Heslop-Harrison 1994; Kondo and Abd El-Twab 2002; Abd El-Twab and Kondo 2003). Analyses of NOR distribution and organization in rDNA carrying chromosomes are important to understand the events of divergence and hybridization of species. Molecular cytogenetic approach to elucidate the species relationships among the Japanese Chrysanthemum species studied were firstly reported by Kondo et al. (1996). Thus, the 45S rDNA probe $\mathrm{pTa} 71$ has promised to mark NOR's of satellites for identification of sat-chromosomes among the chromosome complements in several Japanese 
and Chinese species in Chrysanthemum in broad sense studied (Khaung et al. 1997; Honda et al. 1997; Abd ElTwab et al. 1999; Abd El-Twab and Kondo 1999; Abd El-Twab and Kondo 2003).

In the present work, C. zawadskii showed variation in the morphological characters, therefore it was very interesting to investigate the genome variation within this species. We aimed to produce physical maps of the location of the $5 \mathrm{~S}$ and $45 \mathrm{~S}$ rDNA and telemoric repeats, their chromosomal positions on the somatic chromosomes by single color and simultaneous bicolor of FISH.

\section{Materials ANd Methods}

The plants that were used in this study were Chrysanthemum zawadskii (sn: Russ-13, 27 and 28-2004) are cultivated in the laboratory of Plant Chromosome and Gene Stock, Graduate School of Science, Hiroshima University.

Chromosome preparations Following Kondo et al. (1996) growing root tips were collected and pretreated in $0.002 \mathrm{M} 8$-hydroxyquinolin at $18^{\circ} \mathrm{C}$ for 1.5 hours. They were fixed in the 1: 3 of glacial acetic acid and ethanol at $4^{\circ} \mathrm{C}$ for 2 hours. Fixed roots were excised and washed in distilled water many times to remove the fixative. Five to ten tips $2-5 \mathrm{~mm}$ long each from root tips were placed in $1.5 \mathrm{ml}$ microcentrifuge tube containing the enzymatic buffer of 5\% cellulase (Yakult), 2\% pectolyase Y-23 (Kikkoman). They were incubated at $37^{\circ} \mathrm{C}$ for $20 \mathrm{mins}$, and then, their soft meristematic tissues were washed in distilled water to remove the enzymatic solution and were squashed in $45 \%$ acetic acid. The cover-slip was removed by the dry-ice freezing method and the preparation was dried at room temperature.

5S rDNA probe The 5S rDNA probe was produced by the polymerase chain reaction (PCR) method as described by Hizume (1993) and Abd El-Twab and Kondo (2002).

$45 \mathrm{~S}$ rDNA probe The probe of pTa71 (45S rDNA) consisted of a $9 \mathrm{~kb}$ Eco RI fragment of rDNA derived from Triticum aestivum L. (Gerlach and Bedbrook 1979), which was recloned into pUC19 plasmid. It was comprised of the coding sequence for the $18 \mathrm{~S}, 5.8 \mathrm{~S}$ and $26 \mathrm{~S}$ genes and the non-transcribed spacer sequences.

The probe of the telomere sequence repeats The telomere sequence repeats were purchased and synthesized by using the polymerase chain reaction (PCR). PCR was carried out in the absence of template using primers

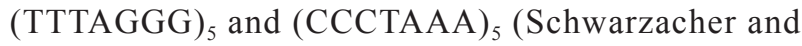
Heslop-Harrison 1991; Cox et al. 1993). Each $50 \mu 1$ reaction mixture include: $50 \mathrm{mM}$ Tris- $\mathrm{HCl}, \mathrm{pH} 9.0$ $\left(25^{\circ} \mathrm{C}\right), 50 \mathrm{mM} \mathrm{KCl}, 0.1 \mathrm{mM}$ EDTA, $0.1 \%$ (v/v) Triton $\mathrm{X}-100,1.25 \mathrm{mM} \mathrm{MgCL}_{2} 2.5$ units of Taq polymerase
(TOYOBO), $200 \mu \mathrm{M}$ each $\mathrm{dNTP}$ and $200 \mathrm{nM}$ each primer. Temperature cycling was performed as follows: The first step of one cycle each for one minute at $94^{\circ} \mathrm{C}$, and 30 seconds at $55^{\circ} \mathrm{C}$ and one minute at $72^{\circ} \mathrm{C}$; the second step of 35 cycles each for 30 seconds at $94^{\circ} \mathrm{C}, 30$ seconds at $55^{\circ} \mathrm{C}$ and one minute at $72^{\circ} \mathrm{C}$; and the final step for three minutes at $72^{\circ} \mathrm{C}$. The product was purified by PCI (phenol: chloroform: isoamilalcohol, 25:24:1) treatment, CIA treatment and ethanol precipitation method. The oligonucleotide purified, the telomere sequence repeats, were dissolved in $5 \mu \mathrm{l}$ of TE buffer $(10.0 \mathrm{mM}$ Tris. $\mathrm{Cl}$ at $\mathrm{pH} 8.0$ and $1.0 \mathrm{mM}$ EDTA at $\mathrm{pH} 8.0$ ). The concentration of the products was measured with the absorptionmeter (Ultrospec 3000). Then, the telomere sequence repeats were used for labeling reaction.

Labeling of the probes The probes were labeled either with biotin-14-dATP nick translation kit (Gibco BRL) or digoxigenin (DIG)-dUTP by Dig DNA labeling kit (Boehringer Mannheim) labeling of each probe was carried out according to the manufacturer's protocols.

The procedure of FISH and detection of the probe followed the methods described by Abd El-Twab and Kondo (1999). Single color of FISH that fluoresced the yellow-color was visualized by FITC after the hybridization with the biotin labeled probes and red-color was visualized by the counter stain of PI. Bicolor of FISH that fluoresced the yellow-color signal that hybridized with biotin labeled probe was visualized by FITC, the red-color signal that hybridized with digoxiginin labeled probe was visualized by rhodamin and the counter stain fluoresced blue-color was visualized by DAPI. The fluorescence signals were examined with an epifluorescence microscope with Nikon B-2A filter cassette and microphotographs were taken on CCD camera (Pixera Pengium $600 \mathrm{CL}$ ). Analysis of hybridization signals and superimposed image was produced using Adobe Photoshop 7.

\section{Results AND Discussion}

Chrysanthemum zawadskii that were collected from Russia during the field trip of 2004 showed chromosome number of $2 \mathrm{n}=54$ (hexaploid) and big variation in the color of the ligules (from white to pinkish). FISH signals on mitotic interphase (Fig. 1. A, C and E) and metaphase (Fig. 1. B, D and F) chromosomes in C. zawadskii. Eight signals of 5S rDNA (A-B; six strong-large and two weak-small signals), which were six interstitial and two terminal, which hybridized with the biotin labeled probe of the PCR amplified 5S rDNA. Fourteen signals of the 45S rDNA (Fig. 1. C-D, ten terminal and four interstitial), which hybridized with the biotin labeled probe of pTa71. Terminal yellow signals of the telomeres (Fig. 1. E-F), which hybridized with the biotin labeled probe of the PCR amplified telomeric repeats. Divergence and speciation are often accompanied by reorganization in the 

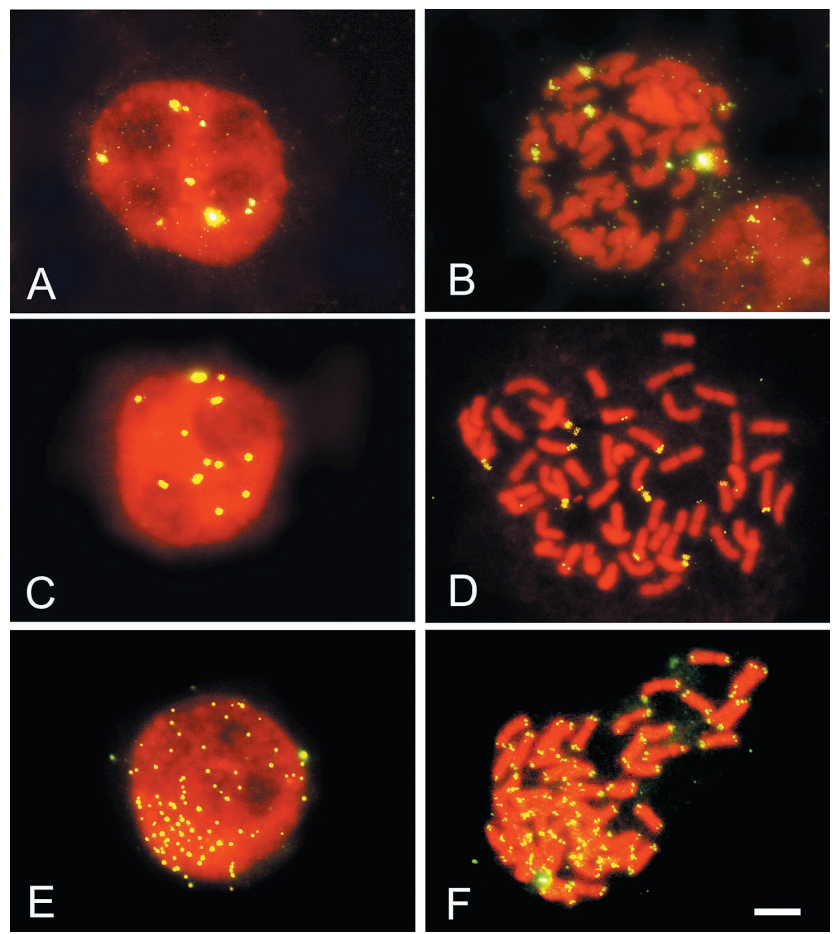

Fig. 1. FISH signals on mitotic interphase (A, C and E) and metaphase (B,D and F) chromosomes in C. zawadskii (sn: Russ-13-, 27- and 28-2004). Yellow-color was fluoresced and visualized by FITC after the hybridization with the biotin labeled probes and red-color was visualized by the counter stain of PI. A-B. Eight signals of 5S rDNA (six strong-large and two weaksmall signals), which hybridized with the biotin labeled probe of the PCR amplified 5S rDNA. C-D. Twelve signals of the 45S rDNA that hybridized with the biotin labeled probe of pTa71. E-F. Terminal yellow signals of the telomeres that hybridized with the biotin labeled probe of the PCR amplified telomeric probe. Bar $=10 \mu \mathrm{m}$.

chromosomes. However, the karyotypes of Chrysanthemum species are highly conserved and it is difficult to differentiate closely related species, such as species of the same ploidy level by karyotyping (Tanaka et al. 1989). In contrast, rDNA FISH gives much higher resolution for detecting chromosome variations among closely related species. Our results and other studies (Kondo et al. 1996; Khaung et al. 1997; Honda et al. 1997; Abd El-Twab and Kondo 2003) have shown that within the genus Chrysanthemum (=Dendranthema) the number and positions of major rDNA sites on chromosomes vary among species. This variation should have phylogenetic implications, since the more similar rDNA FISH patterns are likely to be in the most closely related the taxa (Abd El-Twab and Kondo 2003). The distribution patterns of rDNA loci among the chromosomes vary markedly among Chrysanthemum in broad sense. In diploid species the number of major $45 \mathrm{~S}$ rDNA sites typically ranges between 1 and 4 pairs (e.g. Abd El-Twab and Kondo 2003). The highest percentage FISH signal number of the $45 \mathrm{~S}$ rDNA per chromosome complement among the wild species of Chrysanthemum was $44.4 \%$ in diploid species of $C$. lavandulifolium while the highest percentage among the wild hexalpoid species was 33.3\% in C. occidentali-japonense (Abd El-Twab and Kondo 2003 ) and was $55.5 \%$ in the cultivars of Dendranthema studied up to date (Abd El-Twab and Kondo, submitted). In the present study the percentage of the FISH signal number of the 45S rDNA was approximately 25.9-35.2\%, indicating this C. zawadskii studied up to date has a complex genome.

Since, a prediction of chromosomal locations of satellites would be that closely related satellite sequences show similar chromosomal distributions. Closely related satellite repeats occupy adjacent regions of the chromosomal DNA. Such, topological constraints might arise from either functional restrictions or from mode of origin, one from the other (Lohe and Roberts, 1988). In Chrysanthemum the 5S rDNA arrays tend to be localized at one site on one to three homologous pairs of chromosomes (Abd El-Twab and Kondo 2002). Use of doubleor multiple-probe FISH facilitates the identification of homologous chromosomes and the difference of changes in genome structure among species. It also helps to clarify genetic maps and to assign linkage groups to physically marked chromosomes. By comparing the rDNA FISH patterns identified homologous chromosomes among the species. Analysis of the linkage pattern of the $5 \mathrm{~S}$ and $45 \mathrm{~S}$ rDNA in the present studied taxa revealed chromosomes that harbored loci for both classes of rDNA.

Bicolor FISH signals of $5 \mathrm{~S}$ and $45 \mathrm{~S}$ rDNA in mitoticmetaphase chromosomes in C. zawadskii (Fig. 2). The chromosome complement that counter-stained blue-color (Fig. $2 \mathrm{~A}$ ) showed nineteen signals of $45 \mathrm{~S}$ rDNA stained red-color (Fig. 2 B) and seven signals of $5 \mathrm{~S}$ rDNA stained yellow-green-color (Fig. 2 C). The superimposed image of a combination of Fig. 2. A-C, located the distribution of the yellow $5 \mathrm{~S}$ and red $45 \mathrm{~S}$ rDNA sites (Fig. 2 D). The hybridization sites on the chromosomes could be distinguished in three groups, the first group had only terminal signals of the $45 \mathrm{~S}$ rDNA on the short arm and were 12 chromosomes, the second group was two chromosomes had co-localized-terminal signals (arrowheads) of both of the 5S and 45S rDNA on the short arm, third group was five chromosomes had interstial signals (arrows) of both of the 5S and 45S rDNA.

There are two types of linkage pattern of the $5 \mathrm{~S}$ and $45 \mathrm{~S}$ rDNA, the first type is the co-localization on the same chromosome, which was interstitial in the long arm or in the terminal region of the short arm, these chromosomes are likely homologous. This pattern was observed only in the present species for the first time. The second type was distributed separately on different chromosomes without any co-localization. This pattern is the common type in the wild species of Chrysanthemum. All these findings of the physical mapping of the $5 \mathrm{~S}$ and $45 \mathrm{~S}$ rDNA indicate that the genome of C. zawadskii has undergone changes since its speciation through natural hybridization and genome reorganization. 

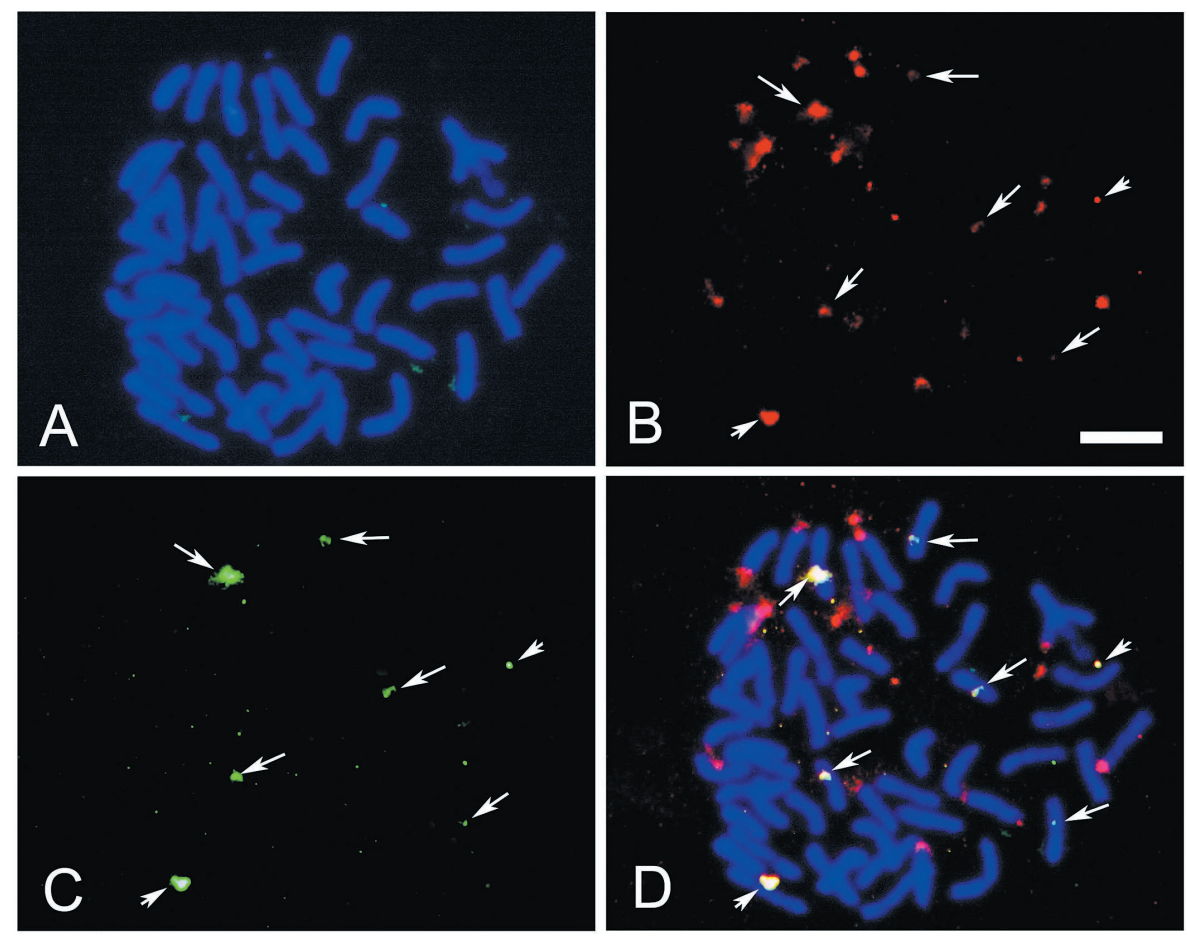

Fig. 2. Bicolor FISH signals of 5S and 45S rDNA in mitotic-metaphase chromosomes in C. zawadskii (sn: Russ-13- and 28-2004). The yellow-color signal that hybridized with biotin labeled probe was fluoresced and visualized by FITC, the red-color signal that hybridized with digoxiginin labeled probe was visualized by rhodamin and the counter stain was blue-color by DAPI. A. A chromosome complement counter-stained blue-color. B. Fourteen signals of 45S rDNA stained red-color. C. Seven signals of 5S rDNA stained yellow-green-color. D. Superimposed image of Superimposed image of a combination of A-C to locate the distribution of the yellow$5 \mathrm{~S}$ and red-45S rDNA sites. The hybridization sites on the chromosomes could be distinguished in three groups, the first group had only terminal signals of the $45 \mathrm{~S}$ rDNA on the short arm and were 10 chromosomes, the second group was two chromosomes had colocalized-terminal signals (arrowheads) of both of the 5S and 45S rDNA on the short arm, third group was five chromosomes had interstial signals (arrows) of both of the $5 \mathrm{~S}$ and $45 \mathrm{~S}$ rDNA. Bar $=10 \mu \mathrm{m}$.

Studies on origin and chromosome constitutions of the native polyploid species of Chrysanthemum in broad sense may contribute to satisfactory phylogenetic and taxonomic treatment of species relationships. In the course of evolution, the species genome appears to have undergone structural reorganization and recombination and, thus, formed unique chromosome complement characteristics as we observed. Knowledge of the relative physical locations and the number of multicopy rDNA gene loci is important and useful both for the construction of physical maps of chromosomes and for phylogenetic studies. Our data of the double FISH provided useful information on the simultaneous presence of both rDNA types as well as their relative position to chromosomes of the species, which is considered very important landmark for identifying specific chromosomes of this species in the artificial produced hybrids in our laboratory.

ACKNOWLEDGMents. This study was supported by the Grantin-Aid for Scientific Research Program (A) No. 10044209 of Japan Society for the Promotion of Science (Representative: Katsuhiko Kondo) and the National Bioresource Project "Chrysanthemum sensu lato" of the Japanese Ministry of Education, Science, Sports and Culture (Representative: Katsuhiko Kondo) This paper was contributed from Laboratory of Plant Chromosome and Gene Stock, Graduate School of Science, Hiroshima University (Contribution No. 93).

\section{Literature Cited}

Abd El-Twab, M. H. and Kondo, K. 2003. Physical mapping of 45S rDNA loci by fluorescent in situ hybridization and Evolution among polyploid Dendranthema species. Chrom. Sci. 7: 71-76.

Abd El-Twab, M. H. and Kondo, K. 2002. Physical mapping of 5S rDNA in chromosomes of Dendranthema by fluorescence in situ hybridization. Chrom. Sci. 6: 13-16.

Abd El-Twab, M. H. and Kondo, K. 1999. Identification of nucleolar organizing regions and parental chromosomes in F1 hybrid of Dendranthema japonica and Tanacetum vulgare simultaneously by fluorescence in situ hybridization. Chrom. Sci. 3: 59-62.

Abd El-Twab, M. H., Kondo, K. and Hong, D. 1999. Isolation of a particular chromosome of Ajania remotipinna in a chromosome complement of an artificial $\mathrm{F}_{1}$ hybrid of Dendranthema lavandulifolia X Ajania remotipinna by use of genomic in situ hybridization. Chrom. Sci. 3: 21-28.

Cox, A.V., Bennett, S. T., Parokonny, A. S., Kenton, A., Callimassia, M. A. and Bennett, M. D. 1993. Comparison of plant telomere locations using a PCR-generated synthetic probe. Ann. Bot. 72: 239-247.

Gerlach, W. L. and Bedbrook, J. R. 1979. Cloning and characterization of ribosomal RNA genes from wheat and barley. Nucleic Acids Res. 7: 1869-1885.

Harrison, G. E. and Heslop-Harrison, J. S. 1995. Centromeric repetitive DNA sequences in the genus Brassica. Theor. Applied Genettics 90: 157-165.

Heslop-Harrison, J. S. 2000. Comparative Genome Organization in Plants: From Sequence and Markers to Chromatin and Chromosomes. Plant Cell 12: 617-635. 
Heslop-Harrison, J. S., Schwarzacher, T., Anamthawat-Jonson, K., Leich, A. R., Shi, M. and Leich, I. J. 1991. In situ hybridization with automated chromosome denaturation. Technique 3: 109-116.

Hizume, M. 1993. Chromosome localization of 5S rDNA genes in Vicia faba and Crepis capillaris. Cytologia 58: 417-421

Honda, Y., Abd El-Twab, M. H., Ogura, H., Kondo, K., Tanaka, R. and Shidahara,T. 1997. Counting sat-chromosome numbers and species characterization in wild species of Chrysanthemum sensu lato by fluorescence in situ hybridization using pTa71 probe. Chrom. Sci. 1: 77-81.

Inafuku, K., Nabeyama, M., Kikuma, Y., Saitoh, J., Kubota, S., and Kohno, S. 2000. Chromosomal location and nucleotide sequence of $5 \mathrm{~S}$ ribosomal DNA of two cyprinid species (Osteichthyes, Pisces). Chrom. Res. 8: 193-199.

Khaung, K., Kondo, K., Tanaka, R. (1997). Physical mapping of rDNA by fluorescent in situ hybridization using pTa71 probe in three tetraploid species of Dendranthema. Chrom. Sci. 1: 25-30.

Kondo, K. and Abd El-Twab, M. H. 2002. Analysis of interageneric relationships sensu stricto among the members of Chrysanthemum sensu lato by using fluorescence in situ hybridization and genomic in situ hybridization. Chrom. Sci. 6: $87-100$.

Kondo, K., Honda, Y. and Tanaka, R. 1996. Chromosome marking in Dendranthema japonica var. wakasaense and its closely related species by fluorescence in situ hybridization using rDNA probe. La Kromosomo II-81: 2785-2791.
Kondo, K., Tanaka, R., Hong, D., Hizume, M., Yang, Q. and Nkata, M. 1994. A chromosome study of Ajania and its allied genera in the Chrysantheminae, the Anthemideae, the Compositae in Chinese highlands. pp. 1-13 In: R. Tanaka Ed., Karyomorphological and cytogenetical studies in plants common to Japan and China. Hiroshima University p: 75.

Leich, I. J. and Bennett, M. D. 1997. Polyploidy in angiosperms. Trends in Plant Science, 2, 12: 470-471.

Lewin, B. 1997. Gene numbers, pp. 687-711. In: Genes. Oxford Univ. Press, p. 1260.

Lohe A. and Roberts P. 1988. The biology of heterochromatin. In Heterochromatin molecular and structural aspects, ed. R. H. Verma, pp 148-186. New York: Cambridge Univ. Press.

Ørgaard, M. and Heslop-Harrison, J. S. 1994. Investigations of genome relationships between Leymus, Psathyrostachys and Hordium inferred by genomic DNA: DNA in situ hybridization. Ann. Bot. 73: 195-203.

Schwarzacher, T., and Heslop-Harrison, J. S., 1991. In situ hybridization to plant telomeres using synthetic oligomers. Genome 34: 317-323.

Soltis, P. S. and Soltis, D. E. 2000. The role of genetic and genomic attributes in the success of polyploids, Proc. Natl. Acad. Sci. USA 97: 7051-7057.

Tanaka, R., Kawasaki, S., Yonezawa, Y., Taniguchi, K. and Ikeda, H. 1989. Cytogenetic studies on wild Chrysanthemum from China V. $\mathrm{F}_{1}$-hybrids of Chrysanthemum lavandulifolium var. sianense X Ch. boreale. Cytologia 54: 365-372. 